\title{
An endogenous tumour-promoting ligand of the human aryl hydrocarbon receptor
}

\author{
Christiane A. Opitz ${ }^{1,2 *}$, Ulrike M. Litzenburger ${ }^{1,2 *}$, Felix Sahm $^{3}$, Martina Ott ${ }^{1,2}$, Isabel Tritschler ${ }^{4}$, Saskia Trump ${ }^{5}$, \\ Theresa Schumacher ${ }^{1,2}$, Leonie Jestaedt ${ }^{6}$, Dieter Schrenk ${ }^{7}$, Michael Weller ${ }^{4}$, Manfred Jugold ${ }^{8}$, Gilles J. Guillemin ${ }^{9}$, \\ Christine L. Miller ${ }^{10}$, Christian Lutz ${ }^{1}$, Bernhard Radlwimmer ${ }^{12}$, Irina Lehmann ${ }^{5}$, Andreas von Deimling ${ }^{3}$, Wolfgang Wick ${ }^{1,13}$ \\ \& Michael Platten ${ }^{1,2}$
}

\begin{abstract}
Activation of the aryl hydrocarbon receptor (AHR) by environmental xenobiotic toxic chemicals, for instance 2,3,7,8-tetrachlorodibenzo-p-dioxin (dioxin), has been implicated in a variety of cellular processes such as embryogenesis, transformation, tumorigenesis and inflammation. But the identity of an endogenous ligand activating the AHR under physiological conditions in the absence of environmental toxic chemicals is still unknown. Here we identify the tryptophan (Trp) catabolite kynurenine (Kyn) as an endogenous ligand of the human AHR that is constitutively generated by human tumour cells via tryptophan-2,3-dioxygenase (TDO), a liver- and neuron-derived Trp-degrading enzyme not yet implicated in cancer biology. TDO-derived Kyn suppresses antitumour immune responses and promotes tumour-cell survival and motility through the AHR in an autocrine/paracrine fashion. The TDO-AHR pathway is active in human brain tumours and is associated with malignant progression and poor survival. Because Kyn is produced during cancer progression and inflammation in the local microenvironment in amounts sufficient for activating the human AHR, these results provide evidence for a previously unidentified pathophysiological function of the AHR with profound implications for cancer and immune biology.
\end{abstract}

Degradation of Trp by indoleamine-2,3-dioxygenases 1 and 2 (IDO1/2) in tumours and tumour-draining lymph nodes inhibits antitumour immune responses ${ }^{1-5}$ and is associated with a poor prognosis in various malignancies ${ }^{6}$. Inhibition of IDO1/2 suppresses tumour formation in animal models ${ }^{1,3}$ and is currently tested in phase I/II clinical trials in patients with cancer ${ }^{7}$. The relevance of Trp catabolism to human tumour formation and progression is, however, as yet unknown.

\section{TDO degrades Trp to Kyn in human brain tumours}

A screen of human cancer cell lines revealed constitutive degradation of Trp and release of high micromolar amounts of Kyn in brain tumour cells, namely glioma cell lines and glioma-initiating cells (GICs), but not human astrocytes (Fig. 1a). IDO1 and IDO2 did not account for the constitutive Trp catabolism in brain tumours (Supplementary Fig. 1a-e). Instead, tryptophan-2,3-dioxygenase (TDO), which is predominantly expressed in the liver (Supplementary Fig. 3a, b) and is believed to regulate systemic $\operatorname{Trp}$ concentrations ${ }^{6}$, was strongly expressed in human glioma cells (Supplementary Fig. 1b) and correlated with Kyn release (Fig. 1b). Pharmacological inhibition or knockdown of TDO blocked Kyn release by glioma cells, whereas knockdown of IDO1 and IDO2 had no effect (Fig. 1c, d and Supplementary Fig. 2a), thus confirming that TDO is the central Trp-degrading enzyme in human glioma cells. In human brain tumour specimens, TDO protein levels increased with malignancy and correlated with the proliferation index (Fig. 1e-g and Supplementary Fig. 2b-d). As described previously ${ }^{8}$, healthy human brain showed only weak TDO staining in neurons (Fig. 1e). TDO expression was not confined to gliomas but was also detected in other cancer types (Supplementary Fig. 3b, c). Lower Trp concentrations were measured in the serum of patients with glioma (Fig. 1h). These may not have translated into increased Kyn levels (Fig. 1h) because Kyn is taken up by other cells and metabolized to quinolinic acid. Indeed, accumulation of quinolinic acid was detected in TDO-expressing glioblastoma tissue (Fig. 1i and Supplementary Fig. 3d).

\section{Effects of TDO-mediated Kyn release on immune cells}

Kyn suppresses allogeneic T-cell proliferation ${ }^{9}$. Allogeneic T-cell proliferation was inversely correlated with Kyn formation by gliomaderived TDO (Fig. 2a and Supplementary Fig. 4a, b). Knockdown of TDO in glioma cells (Supplementary Fig. 4c, d) restored allogeneic $\mathrm{T}$-cell proliferation, and the addition of Kyn to the TDO knockdown cells prevented the restoration of T-cell proliferation (Fig. 2b). The proliferation of $\mathrm{CD}^{+}{ }^{+}$and $\mathrm{CD} 8{ }^{+} \mathrm{T}$ cells stimulated by the T-cell receptor was inhibited by Kyn in a concentration-dependent manner (Supplementary Fig. 4e). In addition, knockdown of TDO resulted in enhanced lysis of glioma cells by alloreactive peripheral blood mononuclear cells (Supplementary Fig. 4f). Finally, decreased infiltration with leukocyte common antigen (LCA)-positive and $\mathrm{CD}^{+}$immune cells was observed in sections of human glioma with high TDO expression in comparison with those with low TDO expression (Fig. 2c), indicating that Kyn formation by TDO may suppress antitumour immune responses. In vivo experiments in immunocompetent mice demonstrated that tumours expressing TDO grew

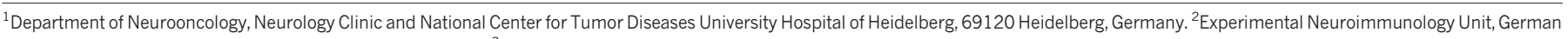

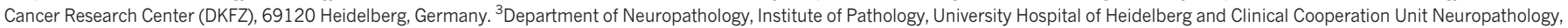

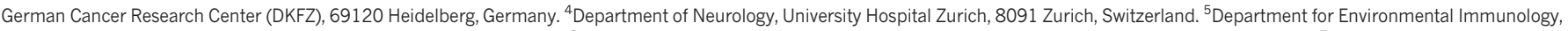

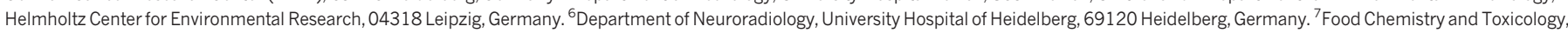

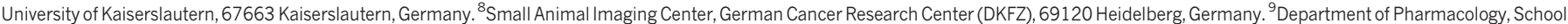

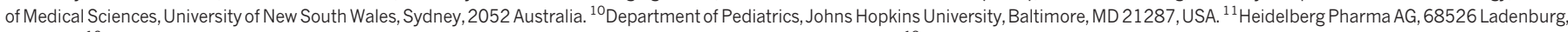

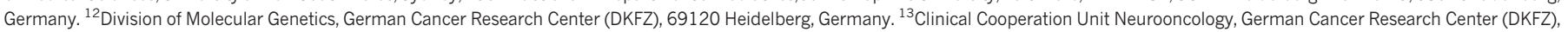
69120 Heidelberg, Germany.

*These authors contributed equally to this work 
a
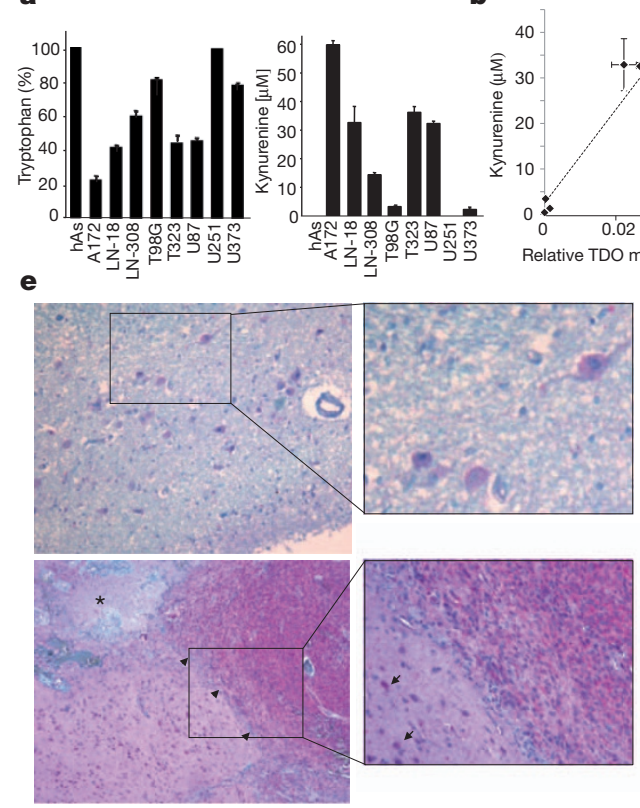

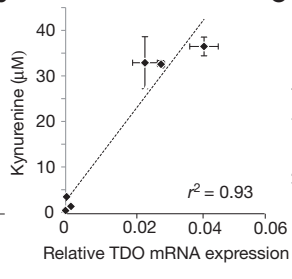

c

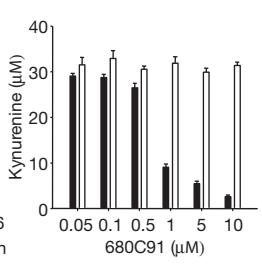

d

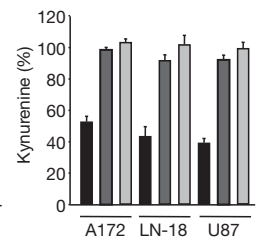

f

$\mathbf{9}$

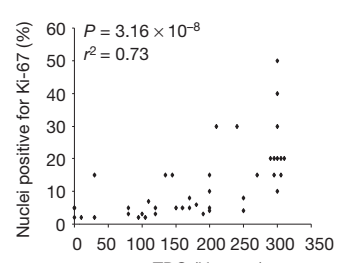

TDO (H-score)

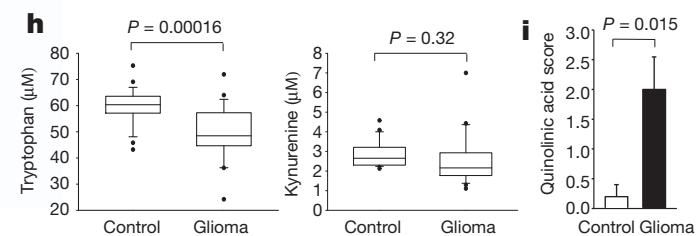

Figure 1 TDO degrades Trp to Kyn in human brain tumours. a, Trp (left) and Kyn (right) content in the supernatants of human astrocytes (hAs), glioma cell lines and GICs (T323) cultured for $72 \mathrm{~h}$ and measured by high-performance liquid chromatography (HPLC) $(n=4)$. b. Correlation between TDO mRNA and Kyn release by human glioma cells measured by quantitative RT-PCR and HPLC $(n=4)$. c, Kyn concentrations in the supernatants of U87 glioma cells cultured for $48 \mathrm{~h}$ in the presence of the TDO inhibitor 680C91 (black bars) or its solvent (white bars; $n=4, P=0.005,0.002$ and 0.0009 for 1,5 and $10 \mu \mathrm{M} 680 \mathrm{C} 91$, respectively). d, Kyn release by glioma cells after knockdown of TDO (black bars; $P=0.000007$, 0.0007 and 0.00006 , respectively), IDO1 (dark grey bars) or IDO2 (light grey bars) by siRNA $(n=3)$. e, Upper panel: weak neuronal TDO expression in healthy brain tissue. Lower panel: TDO expression in glioblastoma (WHO grade IV). TDO staining is in red. Asterisk indicates necrosis; arrowheads indicate the border

faster and had a higher proliferation index than TDO-deficient control tumours (Fig. 2d and Supplementary Fig. 4g-i). TDO activity in tumours suppressed antitumour immune responses in vivo, as demonstrated by a decreased release of interferon- $\gamma$ by tumourspecific $\mathrm{T}$ cells and tumour cell lysis by spleen cells of mice bearing TDO-expressing tumours in comparison with mice bearing TDOdeficient tumours (Fig. 2e, f).

\section{Effects of TDO-mediated Kyn release on glioma cells}

We next assessed the autocrine effects of Kyn on glioma cells. Although no differences in cell cycle progression were detected between controls and glioma cells with TDO knockdown (Supplementary Fig. 5a), knockdown of TDO decreased motility and clonogenic survival (Fig. 2g, h and Supplementary Fig. 5b-d). This was mediated by Kyn because exogenous addition of Kyn restored motility and clonogenic survival in the absence of Trp (Fig. 2i, $j$ and Supplementary Fig. 5e, f), suggesting that Kyn increases the motility of malignant glioma cells. In GICs, sphere formation was enhanced in response to Kyn (Supplementary Fig. 5g). Finally, tumour formation was impaired when TDO knockdown tumours were orthotopically implanted in the brains of nude mice, which are devoid of functional $\mathrm{T}$ cells (Fig. 2k and Supplementary Fig. 5h, i). To analyse whether TDO-mediated inhibition of antitumour natural killer (NK)-cell responses, which are functional in nude mice, might account for the impaired formation of $T D O$ knockdown tumours, we compared subcutaneous tumour growth in the presence or absence of NK cells. NKcell depletion (Supplementary Fig. 5j) enhanced the growth of both control and TDO knockout tumours but did not restore the growth of $T D O$ knockout tumours to that in controls (Fig. 21 and Supplementary Fig. 5k), suggesting that Kyn generated by constitutive TDO activity

to infiltrated brain tissue. Insets: single tumour cells (arrows) infiltrating the adjacent brain tissue. Magnifications: $\times 40$ (main panels), $\times 400$ (upper inset) and $\times 100$ (lower inset). f, Plot of TDO expression ( $H$-score; see Supplementary Methods) in brain tumours of increasing malignancy (WHO grades II-IV; grade II, $n=18$; grade III, $n=15$; grade IV, $n=35$ ). $\mathbf{g}$, Correlation of the Ki-67 proliferative index with the TDO $H$-score in gliomas of different WHO grades $(n=42)$. h, Trp (left) and Kyn (right) concentrations in the sera of 24 patients with glioblastoma and 24 age-matched and sex-matched healthy controls, measured by HPLC. i, Quantification of staining with quinolinic acid in healthy human brain tissue (white bar; $n=5$ ) and glioblastoma tissue (black bar; $n=5$ ). The data distribution in $\mathbf{f}$ and $\mathbf{g}$ is presented as box plots, showing the 25 th and 75 th centiles together with the median; whiskers represent the 10th and 90th centiles, respectively. Error bars indicate s.e.m.

enhances the malignant phenotype of human gliomas in an autocrine manner in the absence of functional antitumour T-cell and NK-cell responses.

\section{Kyn activates the aryl hydrocarbon receptor}

For a better understanding of the molecular mechanisms underlying the autocrine effects of Kyn on glioma cells, we performed microarray analyses of Kyn-treated glioma cells revealing broad induction of aryl hydrocarbon receptor (AHR) response genes by Kyn (Fig. 3a and Supplementary Figs 6a, b and 7). Pathway analyses showed that the 25 genes that were most strongly induced by Kyn treatment in U87 cells at $8 \mathrm{~h}$ and at $24 \mathrm{~h}$ were all directly or indirectly regulated by the AHR (Fig. 3a and Supplementary Fig. 6b). The AHR is a transcription factor of the basic helix-loop-helix (bHLH) Per-Arnt-Sim (PAS) family, which is activated by xenobiotics such as benzo[a]pyrene and 2,3,7,8-tetrachlorodibenzo- $p$-dioxin (TCDD) $)^{10}$. Malignant glioma cell lines and GICs express the AHR constitutively (Supplementary Fig. 6c $)^{11}$, and upregulation of AHR target genes by Kyn was confirmed in two different glioma cell lines (Supplementary Fig. 6d, e). Kyn led to translocation of the AHR into the nucleus after $1 \mathrm{~h}$, thus showing an immediate effect of Kyn on the AHR (Fig. 3b, c and Supplementary Fig. 8a). In accordance with this, western blot analyses of Kynactivated tumour cells showed decreased cytoplasmic localization paralleled by increased nuclear accumulation of the AHR comparable to that induced by TCDD (Fig. 3d). In the nucleus the AHR forms a heterodimer with the AHR nuclear translocator (ARNT) that interacts with the core-binding motif of the dioxin-responsive elements (DRE) located in regulatory regions of AHR target genes ${ }^{12}$. Kyn induced DRE-luciferase activity in glioma cells, with a concentration giving half-maximal response $\left(\mathrm{EC}_{50}\right)$ of $36.6 \mu \mathrm{M}$ (Fig. 3e, 


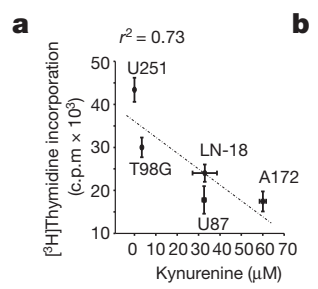

e

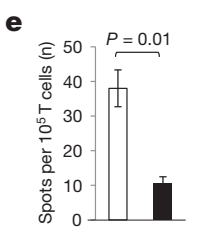

f
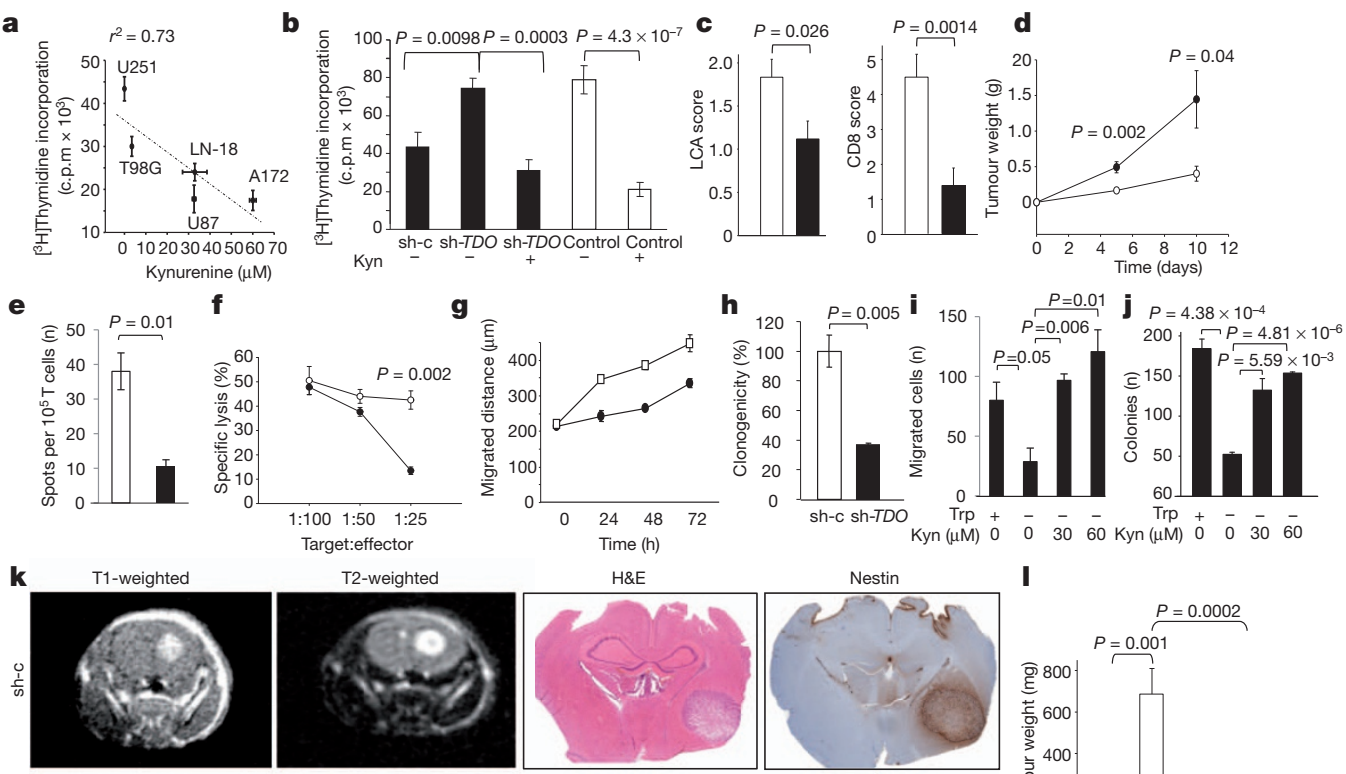

T2-weighted

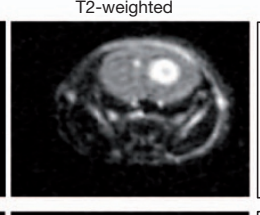

$\mathrm{H} \& \mathrm{E}$ Nestin
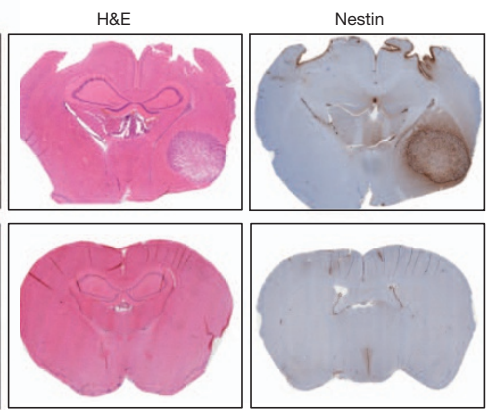

I

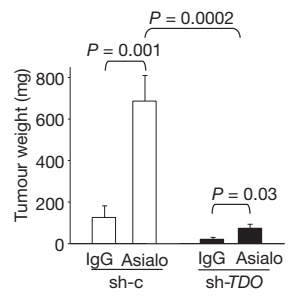

Figure 2 Paracrine and autocrine effects of TDO-mediated Kyn release by glioma cells. a, Correlation of the proliferation of peripheral blood mononuclear cells (PBMCs) cultured with allogeneic glioma cell lines with the Kyn release of the glioma cells $(n=3)$. $\mathbf{b}$, Proliferation of PBMCs cultured with allogeneic TDO-expressing control U87 glioma cells (sh-c) in comparison with U87 glioma cells with a stable short hairpin RNA-mediated knockdown of TDO (sh-TDO), with or without $100 \mu \mathrm{M}$ Kyn (black bars), in comparison with PBMCs alone with or without $100 \mu \mathrm{M}$ Kyn (white bars; $n=3$ ).

c, Quantification of $\mathrm{LCA}^{+}$cells (left) and CD8 ${ }^{+}$cells (right) stained in human glioma sections with low TDO expression ( $H$-score $<150$, white bar; $n=12$ for LCA, $n=10$ for CD8) and in human glioma sections with high TDO expression ( $H$-score $\geq 150$, black bar; $n=17$ for LCA, $n=10$ for CD8). d, Growth of Tdo-deficient GL261 murine glioma cells stably transfected with Tdo (filled circles) or empty vector (open circles) injected subcutaneously into the flank of C57BL/6N mice was monitored using metric callipers $(n=6)$. Tumour weight $(\mathrm{g})$ was calculated as $0.5 \times$ length $(\mathrm{cm}) \times$ width $^{2}\left(\mathrm{~cm}^{2}\right)$. e, Interferon- $\gamma$ release by $\mathrm{T}$ cells of mice bearing subcutaneous Tdo-expressing tumours (black bar) in comparison with T cells of mice bearing Tdo-deficient

Supplementary Fig. 8b). AHR activation was unique to Kyn in a panel of Trp catabolites (Supplementary Table 1). An ethoxyresorufin-Odeethylase (EROD) assay confirmed the induction of the functional AHR target gene CYP1A1, encoding cytochrome $\mathrm{P}_{450}$, family 1 , subfamily A, polypeptide 1 , with an $\mathrm{EC}_{50}$ of $12.3 \mu \mathrm{M}$ for Kyn (Supplementary Fig. 8c). Radioligand binding assays with mouse liver cytosol from $A h r$-proficient and Ahr-deficient mice showed that Kyn binds to the AHR with an apparent $K_{\mathrm{d}}$ of roughly $4 \mu \mathrm{M}$ (Fig. $3 \mathrm{f}$ ).

Activation of the AHR and upregulation of AHR-regulated gene expression in response to Kyn were inhibited by the AHR antagonist dimethoxyflavone or knockdown of AHR (Fig. 3g and Supplementary Fig. $8 \mathrm{~g}-\mathrm{k}$ ), indicating that Kyn is a specific agonist of the AHR. The involvement of the same or similar AHR residues in the binding to Kyn, TCDD and 3-methylcholanthrene was confirmed by the fact that dimethoxyflavone inhibited the activation of the AHR by all three ligands (Supplementary Fig. 8g-i).

The effects of TDO-derived Kyn are mediated by the AHR The endogenous production of Kyn in glioma cells was sufficient to activate the AHR, because knockdown of TDO decreased the expression of AHR-regulated genes (Fig. 3h and Supplementary Fig. 8l-o).

tumours (white bar) after re-stimulation with glioma lysates measured by ELISpot $(n=3)$. f, Lysis of GL261 murine glioma cells by spleen cells of mice with Tdo-expressing GL261 tumours in comparison with those with subcutaneous Tdo-deficient GL261 tumours, measured by chromium release $(n=4)$. g, Quantification of the migrated distances of sh-c (open squares) and sh-TDO (filled circles) cells into a collagen matrix $(n=3, P=0.004,0.0005$ and 0.01 for 24,48 and $72 \mathrm{~h}$, respectively). h, Clonogenic survival of sh-c (white bar) and sh-TDO (black bar) U87 cells $(n=3)$. i, Matrigel Boyden chamber assay of U87 glioma cells in the absence or presence of $70 \mu \mathrm{M}$ Trp without or with 30 or $60 \mu \mathrm{M}$ Kyn $(n=3)$. j, Clonogenic survival of LN-18 glioma cells in the absence or presence of $70 \mu \mathrm{M}$ Trp without or with 30 or $60 \mu \mathrm{M} \mathrm{Kyn}(n=3)$. k, Representative cranial MRIs, haematoxylin/eosin staining ( $\mathrm{H} \& \mathrm{E})$ and nestin staining of CD1 nu/nu mice implanted with sh-c (upper panels) or sh-TDO (lower panels) U87 glioma cells. The images are representative of two independent experiments $(n=6)$. 1, Tumour weight of sh-c (white bars) and sh-TDO (black bars) U87 glioma cells injected subcutaneously in the flank of CD1 nu/nu mice that were treated either with anti-asialo GM1 antibody (asialo) for NK-cell depletion or control IgG (IgG) $(n=8)$. Error bars indicate s.e.m.

Because mean Kyn concentrations of $37.01 \pm 13.4 \mu \mathrm{M}$ were measured in U87 xenografts $(n=6)$, sufficient Kyn concentrations to activate the AHR were also reached in vivo.

In accordance with activation of the AHR by TDO-derived Kyn, expression of the AHR target gene TIPARP in $\mathrm{LCA}^{+}$immune cells was observed only in human glioma sections expressing TDO (Fig. 4a). To determine whether TDO influences antitumour immune responses through the AHR we analysed the infiltration of immune cells in human glioma sections in relation to their AHR expression. Indeed, infiltration by $\mathrm{LCA}^{+}$and $\mathrm{CD}^{+}{ }^{+}$immune cells was decreased in sections of human gliomas with high AHR expression compared with those with low AHR expression (Fig. 4b). To analyse the contribution of host AHR expression to tumour growth, we compared the growth of murine tumours with and without Tdo expression in Ahrdeficient and Ahr-proficient mice. The growth of Tdo-expressing tumours was attenuated in $A h r$-deficient mice when compared with Ahr-proficient mice (Fig. 4c) indicating that AHR-mediated host effects enhance tumour growth. Staining of $\mathrm{LCA}^{+}$immune cells in the tumours revealed that expression of TDO decreased the infiltration with $\mathrm{LCA}^{+}$immune cells in $A h r$-proficient mice but not in $\mathrm{Ahr}$ deficient mice (Fig. 4d and Supplementary Fig. 9a), suggesting that 

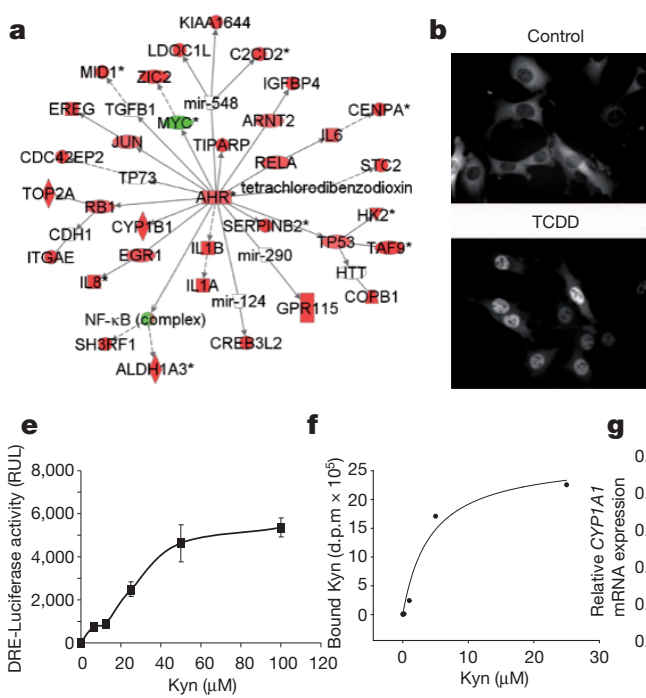

Figure $3 \mid$ Kyn activates the AHR. a, Connection of the 25 genes that were most strongly induced by Kyn treatment in U87 cells after $8 \mathrm{~h}$ to AHR signalling (red, upregulation; green, downregulation). b, Translocation of green fluorescent protein (GFP)-tagged AHR into the nucleus of mouse hepatoma cells, which do not degrade Trp, after $3 \mathrm{~h}$ treatment with $50 \mu \mathrm{M}$ Kyn, $50 \mu \mathrm{M}$ Trp or $1 \mathrm{nM}$ TCDD (negative control: medium). c, Ratios of nuclear to cytoplasmic fluorescent intensity in cells with GFP-tagged AHR after $3 \mathrm{~h}$ of indicated treatment (negative control: medium; positive control: $1 \mathrm{nM}$ TCDD, $50 \mu \mathrm{M}$ Kyn). The data distribution is represented by box plots, showing the 25th and 75th centiles together with the median; whiskers represent the 10th and 90th centiles, respectively $(P<0.001$, one-way analysis of variance on ranks, followed by Dunn's method). d, AHR western blots of two different nuclear $(\mathrm{N})$ and cytoplasmic (C) fractions each of control (lanes 1 and 2), Kyn-treated (lanes 3

TDO-mediated suppression of antitumour immune responses through the AHR contributes to the host effects enhancing the growth of Tdo-expressing tumours. In addition, while in Ahr-proficient mice the expression of Tdo strongly enhanced tumour growth in comparison with tumours not expressing Tdo, the same effect was
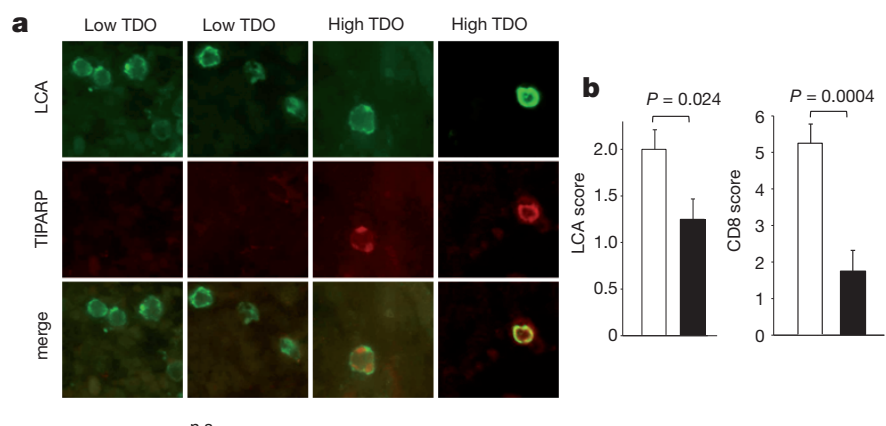

c
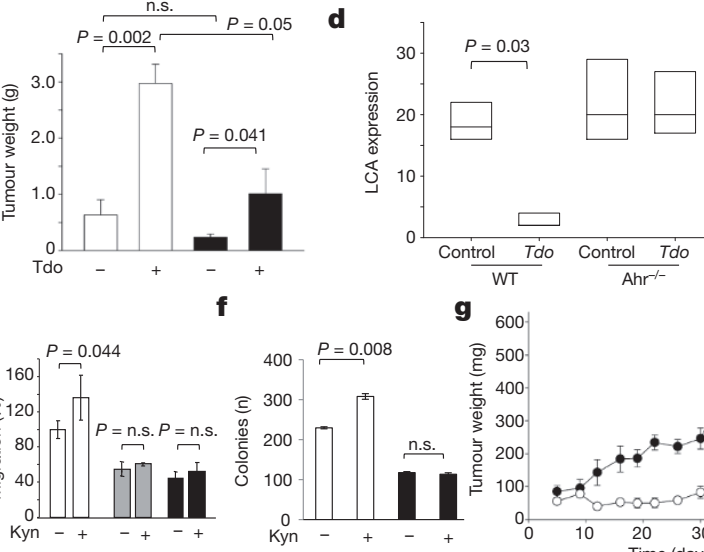

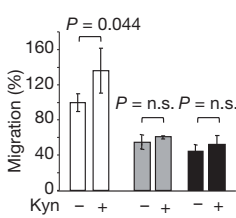

$\mathbf{f}$

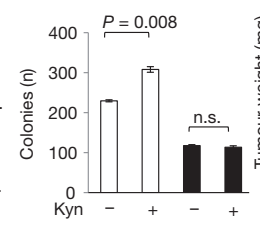

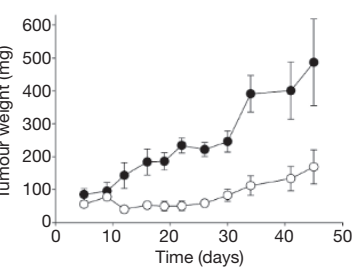

$\operatorname{Trp}$

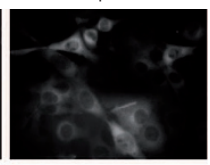

Kyn

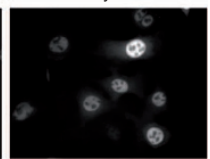

(1)

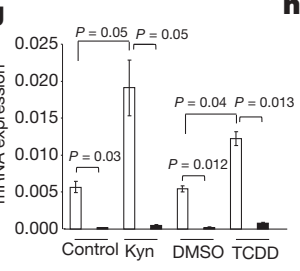

$\mathbf{h}$
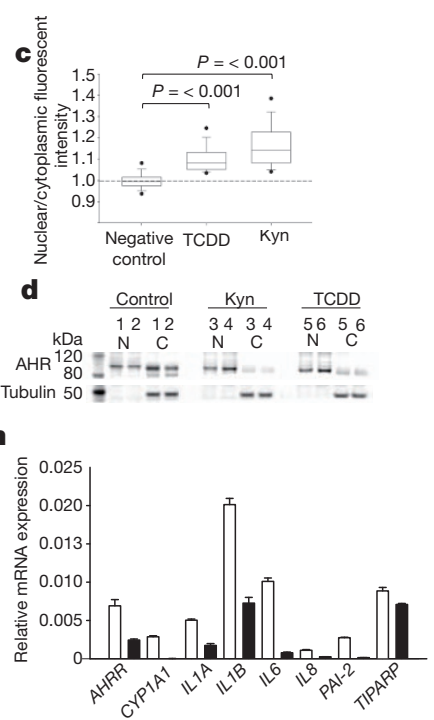

and 4) and TCDD-treated (lanes 5 and 6) human LN-229 glioma cells. e, Dioxinresponsive element (DRE) chemical activated luciferase gene expression in U87 glioma cells treated with the indicated Kyn concentrations $(n=2)$.

f, Radioligand binding assay with indicated concentrations of $\mathrm{L}-\left[{ }^{3} \mathrm{H}\right] \mathrm{Kyn}$ using mouse liver cytosol from $A h r$-proficient and $A h r$-deficient mice. Specific binding was calculated by subtracting the radioactivity measured in $A h r-$ deficient cytosol from that of $A h r$-proficient cytosol $(n=4)$. g, CYP1A1 mRNA expression in sh-AHR LN-308 glioma cells (black bars) in comparison with controls (sh-c; white bars) treated with $100 \mu \mathrm{M}$ Kyn, $1 \mathrm{nM}$ TCDD or controls $(n=4)$. h, mRNA expression of AHR target genes in sh-TDO (black bars) in comparison with sh-c U87 glioma cells (white bars; $n=4)(A H R R, P=0.02$; CYP1A1, $P=0.0007$; IL1A, $P=0.001$; IL1B, $P=0.0000006$; IL6, $P=0.0047$; IL8, $P=0.01$; PAI-2, $P=0.0005$; TIPARP, $P=0.06)$. Error bars indicate s.e.m.

observed in Ahr-deficient mice, although to a much smaller extent (Fig. 4c). Because murine glioma cells express functional AHR (Supplementary Fig. 9b-e), these results suggest that the increase in tumour growth mediated by TDO in Ahr-deficient mice is due to autocrine effects of TDO on the tumour cells themselves.

This notion is supported by the fact that Kyn failed to induce motility of human glioma cells after $A H R$ knockdown (Fig. 4e). In addition, the increase in clonogenic survival in response to Kyn was abolished in glioma cells with a knockdown of $A H R$ (Fig. 4f). Finally,

Figure $4 \mid$ The autocrine and paracrine effects of TDO-derived Kyn are mediated through the AHR. a, Immunofluorescence staining of LCA and TIPARP in human glioma sections with low or high TDO expression. Magnification $\times 400$. b, Quantification of $\mathrm{LCA}^{+}$cells (left) and $\mathrm{CD}^{+}$cells (right) stained in human glioma sections with low AHR expression (Histoscore $<150$, white bar; $n=10$ for LCA, $n=8$ for CD8) and in human glioma sections with high AHR expression ( $H$-score $\geq 150$, black bar; $n=12$ for LCA, $n=12$ for CD8). c, Tumour weight measured 15 days after subcutaneous injection of murine GL261 glioma cells with and without Tdo expression in the flanks of $A h r$-proficient mice (white bars) or Ahr-deficient mice (black bars; $n=6$ ). d, Quantification of $\mathrm{LCA}^{+}$immune cells stained in the subcutaneous Tdo-proficient and Tdo-deficient GL261 tumours in Ahrproficient and Ahr-deficient mice presented as box plots, showing the 25th and 75 th centiles and the median $(n=4)$. WT, wild type. e, Migration of sh-c LN308 glioma cells (white bars) and LN-308 glioma cells with knockdown of the $A H R$ by two different shRNAs (grey bars, sh- $A H R 1$; black bars, sh- $A H R 2$ ) in the presence or absence of $100 \mu \mathrm{M} \mathrm{Kyn}(n=4)$. f, Clonogenicity of sh-c (white bars) and sh-AHR (black bars) LN-308 glioma cells with or without $100 \mu \mathrm{M}$ Kyn $(n=3)$. g, Growth of $A H R$-proficient (filled circles) and AHR-deficient (open circles) human LN-308 glioma cells injected subcutaneously into the flank of $C D 1 n u / n u$ mice was monitored with metric callipers $(n=7)$. Tumour weight was calculated as in Fig. 2d. $P$ values: day 5, 0.147; day 9, 0.546; day 12, 0.027 ; day $16,0.008$; day $19,7.18 \times 10^{-4}$; day $22,1.77 \times 10^{-5}$; day 26 , $1.57 \times 10^{-5}$; day $30,8.49 \times 10^{-4}$; day $34,8.26 \times 10^{-4}$; day $41,0.022$; day 45 , 0.0477. Error bars indicate s.e.m. 
in vivo experiments demonstrated that induced knockdown of $A H R$ in human glioma cells inhibited tumour growth in immunocompromised mice (Fig. 4g and Supplementary Fig. 9f), underscoring the importance of AHR signalling for the autocrine effects of Trp degradation.

\section{TDO-derived Kyn activates the AHR in human cancer}

Next we aimed at investigating whether TDO-derived Kyn activates the AHR in human brain tumour tissue. Indeed, TDO expression correlated with the expression of the AHR and AHR target genes in human glioma tissue (Fig. 5a-c and Supplementary Fig. 9g), indicating that constitutive TDO expression in glioma cells produced sufficient Kyn levels to activate the AHR. To address whether the TDO-Kyn-AHR signalling pathway is also activated in cancers other than glioma, we analysed microarray data of diverse human tumour entities. Interestingly, TDO expression correlated with the expression of the AHR target gene CYP1B1 not only in glioma (Fig. 5c) but also in B-cell lymphoma, Ewing sarcoma, bladder carcinoma, cervix carcinoma, colorectal carcinoma, lung carcinoma and ovarian carcinoma (Fig. 5d, Supplementary Fig. 10a and Supplementary Table 2). This finding indicates that the TDO-KynAHR pathway is not confined to brain tumours but seems to be a common trait of cancers. Analysis of the Rembrandt database revealed that the overall survival of patients with glioma (WHO grades II-IV) with high expression of TDO, the AHR or the AHR target gene CYP1B1 was reduced in comparison with patients with intermediate or low expression of these genes (Fig. 5e and Supplementary Fig. 10b) ${ }^{13}$. Finally, in patients with glioblastoma (WHO grade IV) $)^{14}$, the expression of the AHR targets CYP1B1, IL1B, IL6 and IL8, which are regulated by TDO-derived Kyn in glioma cells (Fig. $3 \mathrm{~h}$ and Supplementary Fig. 5d, e), were found to predict survival independently of WHO grade (Fig. $5 \mathrm{f}$ and Supplementary Fig. 10c), thus further underscoring the importance of AHR activation for the malignant phenotype of gliomas. In summary, these data suggest that endogenous tumour-derived Kyn activates the AHR in an autocrine/paracrine fashion to promote tumour progression (Fig. 5g).

\section{Discussion}

Cancer-associated immunosuppression by Trp degradation has until now been attributed solely to the enzymatic activity of IDO in cancer cells and tumour-draining lymph nodes. IDO inhibition is therefore currently being evaluated as a therapeutic strategy in the treatment of cancer in clinical trials ${ }^{7}$ despite some off-target effects on human cancer cells ${ }^{15}$. We show that TDO is strongly expressed in cancer and is equally capable of producing immunosuppressive Kyn. In IDO-negative glioma cells, TDO seems to be the sole determinant of constitutive Trp degradation, indicating that TDO represents a novel therapeutic target in glioma therapy. In fact, an orally available TDO inhibitor has recently been developed ${ }^{16}$. Inhibition of TDO may not only restore antitumour immune responses but also act on the tumour cell intrinsic malignant phenotype, because we delineated the importance of constitutive Trp degradation to sustain the malignant phenotype of cancer by acting on the tumour cells themselves.

Emerging evidence indicates a tumour-promoting role of the AHR. AHR activation promotes clonogenicity and invasiveness of cancer cells ${ }^{11,17}$. Transgenic mice with a constitutively active AHR spontaneously develop tumours ${ }^{18,19}$, and the repressor of the AHR (AHRR) represents a tumour suppressor in multiple human cancers ${ }^{20}$. The aberrant phenotype of Ahr-deficient mice points to the existence of endogenous AHR ligands ${ }^{21}$. Although different endogenously produced metabolites such as arachidonic acid metabolites, bilirubin, cyclic AMP, tryptamine and 6-formylindolo[3,2-b]carbazole (FICZ) have been shown to be agonists of the $\mathrm{AHR}^{22}$, their functionality has not been convincingly demonstrated in a pathophysiological context such as cancer or immune activation. The search for endogenous ligands of the AHR is therefore continuing.
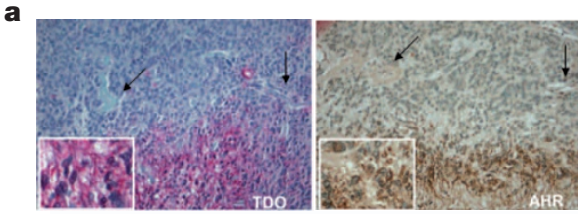

d
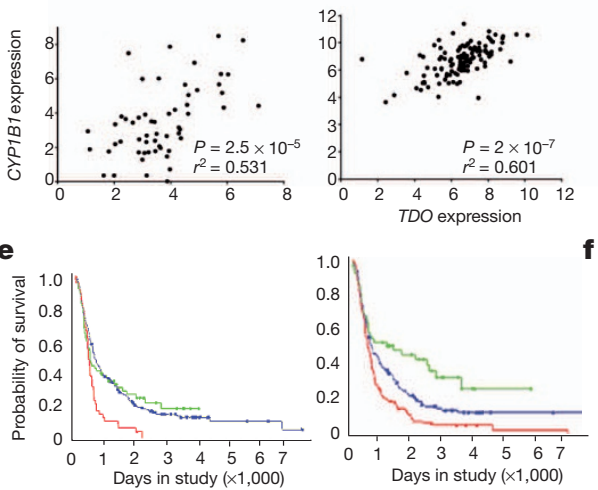

Figure 5 TDO-derived Kyn activates the AHR in diverse human cancers, and AHR activation predicts survival in patients with glioma. a, Correlation of TDO expression (red) and AHR expression (brown) in consecutive sections of human glioblastoma tissue. Arrows indicate vessels for orientation. Magnification $\times 40$; insets $\times 200$. $\mathbf{b}$, Correlation between TDO and AHR expression in human glioma tissue based on $H$-scores of TDO and AHR, calculated by Spearman rank correlation $(n=26)$. c, Correlation between TDO and CYP1B1 expression in microarray data of human glioblastoma $(n=396)$ analysed by Spearman rank correlation. d, Correlation between TDO and CYP1B1 expression in microarray data of human bladder cancer (left; $n=58$ ), human lung cancer (centre; $n=122$ ) and human ovarian carcinoma (right;
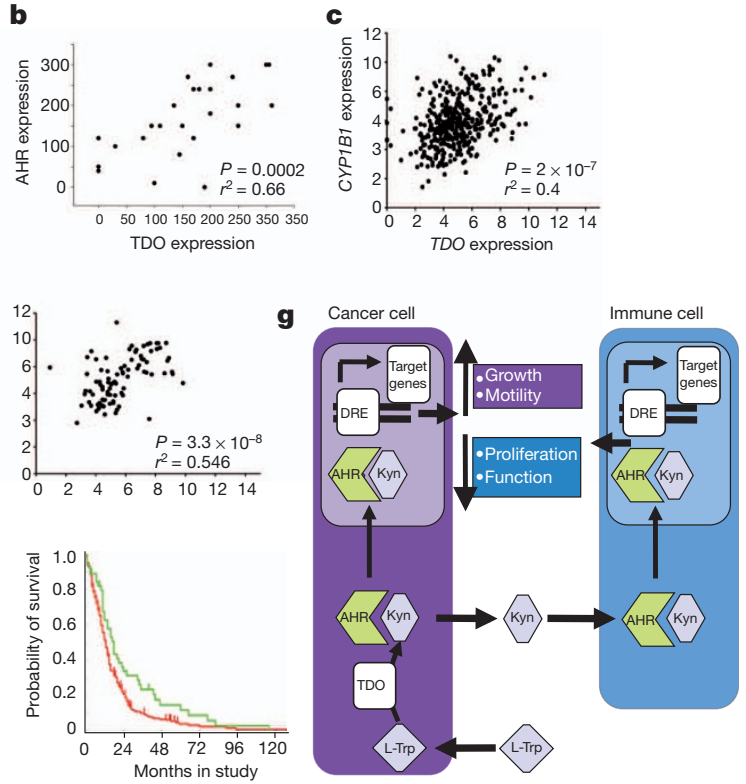

$n=91$ ) analysed by Spearman rank correlation. e, Survival probabilities of patients with glioma (WHO grades II-IV) with high expression (red) of TDO (left) or $A H R$ (right) compared with those of patients with intermediate (blue) or low (green) expression of these genes derived from Rembrandt ${ }^{13}$. f, Survival probabilities of patients with glioblastoma with high expression (red) of the AHR target gene CYP1B1 compared with those of patients with low (green) expression of $C Y P 1 B 1$ derived from the glioblastoma data set of the Cancer Genome Atlas network ${ }^{14}(n=362)$. g, Synoptic figure highlighting the autocrine and paracrine effects of TDO-derived Kyn on cancer cells and immune cells through the AHR. 
We now link these two important pathways contributing to cancer progression by showing that Trp catabolism leads to AHR activation, and we provide evidence of a pathophysiological human condition that is associated with the production of sufficient amounts of a functionally relevant endogenous AHR ligand. Our results reveal a differential response of primary immune cells and transformed cancer cells to AHR-mediated signals, which is in line with various toxicological studies using the classical exogenous AHR ligands TCDD and 3-methylcholanthrene $e^{11,17,23}$. Exposure to these xenobiotics leads to profound suppression of cellular and humoral immune responses ${ }^{23}$, while also promoting carcinogenesis and inducing tumour growth ${ }^{11,17}$. These cell-specific differences in AHR effects are likely to depend on the expression of factors differentially regulating AHR signal transduction such as the $\mathrm{AHRR}^{20}$ as well as cell-specific transcription factor crosstalk shaping the response to AHR activation ${ }^{24}$.

It is likely that Kyn-mediated activation of the AHR is not only relevant in the setting of cancer. For instance, activation of the mouse and human AHR by agonistic ligands induces regulatory $\mathrm{T}$ cells $\mathrm{s}^{25-28}$. Mice with a poor-affinity AHR suffer from exacerbated autoimmune encephalomyelitis in the absence of an exogenous ligand ${ }^{26}$, and Trp catabolites suppress autoimmune neuroinflammation ${ }^{29,30}$, suggesting that activation of Trp catabolism represents an endogenous feedback loop to restrict inflammation through the AHR. In fact, exogenous Kyn is involved in the regulation of immune cells in mice through the $\mathrm{AHR}^{31,32}$. Kyn concentrations sufficient to activate the AHR are also generated by IDO in response to inflammatory stimuli (Supplementary Fig. $11 \mathrm{a}-\mathrm{c}$ ). In a broader context, a significant number of malignancies arise from areas of mostly chronic infection and inflammation ${ }^{33}$, where Trp catabolism in the tumour microenvironment is activated and sustains local immune suppression ${ }^{34}$. Activation of the AHR by Kyn generated in response to inflammatory stimuli may thus constitute a previously unrecognized pathway connecting inflammation and carcinogenesis.

\section{METHODS SUMMARY}

TDO expression was analysed by immunohistochemistry in human tumours. It relevance for Trp degradation was determined by genetic knockdown or overexpression of TDO. Trp and Kyn were measured in cell culture supernatants, human sera and xenograft tissue by high-performance liquid chromatography. Mixed leukocyte reactions, chromium release, ELISpot and staining of immune cells in tumour tissues were used to assess the immune effects of TDO activity. Cell cycle analysis, Matrigel and spheroid invasion assays, scratch assays, sphere formation assays and clonogenicity assays were employed to analyse the autocrine effects of TDO activity. All animal procedures followed the institutional laboratory animal research guidelines and were approved by the governmental authorities. Orthotopic implantation of human glioma cells with and without stable knockdown of TDO into CD1nu/nu mice, subcutaneous injection of these cells into NK-depleted or wild-type $C D 1 n u / n u$ mice and subcutaneous injection of murine Tdo-proficient and Tdo-deficient GL261 cells into syngeneic C57BL/ $6 \mathrm{~N}$ mice were performed to analyse the autocrine and paracrine effects of TDO activity in vivo. Microarray analysis of Kyn-treated human glioma cells was performed to identify signalling pathways activated by Kyn. Analysis of AHR translocation, DRE-luciferase assays and radioligand binding assays confirmed activation of the AHR by Kyn. Pharmacological inhibition and stable knockdown of the AHR (in vitro and in vivo) proved that the effects of Kyn are AHR dependent. Injection of Tdo-proficient and Tdo-deficient tumour cells into $\mathrm{Ahr}^{+/+}$and $A h r^{-1-}$ mice was used to address the contribution of host effects to TDOmediated cancer promotion. Finally, stainings, microarray data and clinical data on human tumour tissues were used to analyse whether TDO activates the AHR in human cancers and how this affects survival.

Received 17 November 2010; accepted 17 August 2011. Published online 5 October 2011.

1. Muller, A. J. et al. Inhibition of indoleamine 2,3-dioxygenase, an immunoregulatory target of the cancer suppression gene Bin1, potentiates cancer chemotherapy. Nature Med. 11, 312-319 (2005).

2. Munn, D. H. \& Mellor, A. L. Indoleamine 2,3-dioxygenase and tumor-induced tolerance. J. Clin. Invest. 117, 1147-1154 (2007).
3. Uyttenhove, C. et al. Evidence for a tumoral immune resistance mechanism based on tryptophan degradation by indoleamine 2,3-dioxygenase. Nature Med. 9, 1269-1274 (2003).

4. Ball, H. J. et al. Characterization of an indoleamine 2,3-dioxygenase-like protein found in humans and mice. Gene 396, 203-213 (2007).

5. Metz, R. et al. Novel tryptophan catabolic enzyme IDO2 is the preferred biochemical target of the antitumor indoleamine 2,3-dioxygenase inhibitory compound D-1-methyl-tryptophan. Cancer Res. 67, 7082-7087 (2007).

6. Lob, S. etal. Inhibitors of indoleamine-2,3-dioxygenase for cancer therapy: can we see the wood for the trees? Nature Rev. Cancer 9, 445-452 (2009).

7. NewLink Genetics Corporation. IDO Inhibitor Study for Relapsed or Refractory Solid Tumors (D-1MT) <http://clinicaltrials.gov/show/NCT00739609> (2008).

8. Miller, C. L. et al. Expression of the kynurenine pathway enzyme tryptophan 2,3dioxygenase is increased in the frontal cortex of individuals with schizophrenia. Neurobiol. Dis. 15, 618-629 (2004).

9. Frumento, G. etal. Tryptophan-derived catabolites are responsible for inhibition of T and natural killer cell proliferation induced by indoleamine 2,3-dioxygenase. J. Exp. Med. 196, 459-468 (2002)

10. Denison, M. S. \& Nagy, S. R. Activation of the aryl hydrocarbon receptor by structurally diverse exogenous and endogenous chemicals. Annu. Rev. Pharmacol. Toxicol. 43, 309-334 (2003).

11. Gramatzki, D. et al. Aryl hydrocarbon receptor inhibition downregulates the TGF- 3 /Smad pathway in human glioblastoma cells. Oncogene $\mathbf{2 8 , 2 5 9 3 - 2 6 0 5}$ (2009).

12. Reyes, H., Reisz-Porszasz, S. \& Hankinson, O. Identification of the Ah receptor nuclear translocator protein (Arnt) as a component of the DNA binding form of the Ah receptor. Science 256, 1193-1195 (1992).

13. National Cancer Institute. REMBRANDT Home Page 〈http:// rembrandt.nci.nih.gov> (2005)

14. The Cancer Genome Atlas Research Network. Comprehensive genomic characterization defines human glioblastoma genes and core pathways. Nature 455, 1061-1068 (2008).

15. Opitz, C. A. et al. The indoleamine-2,3-dioxygenase (IDO) inhibitor 1-methyl-Dtryptophan upregulates IDO1 in human cancer cells. PLOS ONE 6, e19823 (2011)

16. Dolusic, E. et al. Tryptophan 2,3-dioxygenase (TDO) inhibitors. 3-(2(pyridyl)ethenyl)indoles as potential anticancer immunomodulators. J. Med. Chem. 54, 5320-5334 (2011)

17. Bui, L. C.etal. Nedd9/Hef1/Cas-L mediates the effects of environmental pollutants on cell migration and plasticity. Oncogene 28, 3642-3651 (2009).

18. Andersson, P. et al. A constitutively active dioxin/aryl hydrocarbon receptor induces stomach tumors. Proc. Natl Acad. Sci. USA 99, 9990-9995 (2002).

19. Moennikes, 0 . et al. A constitutively active dioxin/aryl hydrocarbon receptor promotes hepatocarcinogenesis in mice. Cancer Res. 64, 4707-4710 (2004).

20. Zudaire, E. et al. The aryl hydrocarbon receptor repressor is a putative tumor suppressor gene in multiple human cancers. J. Clin. Invest. 118, 640-650 (2008).

21. Fernandez-Salguero, P. et al. Immune system impairment and hepatic fibrosis in mice lacking the dioxin-binding Ah receptor. Science 268, 722-726 (1995).

22. Nguyen, L. P. \& Bradfield, C. A. The search for endogenous activators of the aryl hydrocarbon receptor. Chem. Res. Toxicol. 21, 102-116 (2008).

23. Esser, C., Rannug, A. \& Stockinger, B. The aryl hydrocarbon receptor in immunity. Trends Immunol. 30, 447-454 (2009).

24. Frericks, M., Burgoon, L. D., Zacharewski, T. R. \& Esser, C. Promoter analysis of TCDD-inducible genes in a thymic epithelial cell line indicates the potential for cellspecific transcription factor crosstalk in the AhR response. Toxicol. Appl. Pharmacol. 232, 268-279 (2008).

25. Apetoh, L. etal. The aryl hydrocarbon receptor interacts with c-Maf to promote the differentiation of type 1 regulatory T cells induced by IL-27. Nature Immunol. 11, 854-861 (2010)

26. Quintana, F. J. et al. Control of $T_{\text {reg }}$ and $T_{H} 17$ cell differentiation by the aryl hydrocarbon receptor. Nature $\mathbf{4 5 3}, 65-71$ (2008).

27. Quintana, F. J. et al. An endogenous aryl hydrocarbon receptor ligand acts on dendritic cells and $T$ cells to suppress experimental autoimmune encephalomyelitis. Proc. Natl Acad. Sci. USA 107, 20768-20773 (2010).

28. Veldhoen, M. et al. The aryl hydrocarbon receptor links $T_{H} 17$-cell-mediated autoimmunity to environmental toxins. Nature 453, 106-109 (2008).

29. Platten, M. et al. Treatment of autoimmune neuroinflammation with a synthetic tryptophan metabolite. Science 310, 850-855 (2005)

30. Opitz, C. A., Wick, W., Steinman, L. \& Platten, M. Tryptophan degradation in autoimmune diseases. Cell. Mol. Life Sci. 64, 2542-2563 (2007).

31. Mezrich, J. D. et al. An interaction between kynurenine and the aryl hydrocarbon receptor can generate regulatory T cells. J. Immunol. 185, 3190-3198 (2010).

32. Nguyen, N. T. et al. Aryl hydrocarbon receptor negatively regulates dendritic cell immunogenicity via a kynurenine-dependent mechanism. Proc. Natl Acad. Sci. USA 107, 19961-19966 (2010).

33. Coussens, L. M. \& Werb, Z. Inflammation and cancer. Nature 420, 860-867 (2002).

34. Muller, A. J. et al. Chronic inflammation that facilitates tumor progression creates local immune suppression by inducing indoleamine 2,3 dioxygenase. Proc. Natl Acad. Sci. USA 105, 17073-17078 (2008).

Supplementary Information is linked to the online version of the paper at www.nature.com/nature.

Acknowledgements We thank K. Rauschenbach, J. Reiert, S. Koch and A. Mlitzko for technical assistance, P.-N. Pfenning for help with the invasion assays, J. Blaes for help generating genetically modified cells, T.V. Lanz and I. Oezen for help with animal experiments, C. Esser for providing Ahr ${ }^{-1-}$ mice, M. Schwarz for providing the human DRE-luciferase construct, D. Lemke for generating GICs, K. Ochs for providing human 
umbilical-vein endothelial cell cDNA, A. Hertenstein for generation of $\mathrm{CD} 4^{+}$and $\mathrm{CD} 8^{+} \mathrm{T}$ cells, M. Betts and R. B. Russel for modelling of the binding of Kyn to the AHR, R. Koch for performing quantitative reverse transcriptase polymerase chain reaction analyses,

R. Tudoran for generation of GL261 cells overexpressing murine TDO, M. Deponte for help with the radioligand binding assay, D. Schemmer for collecting and banking serum samples, W. Roth for providing tissue specimens, M. Remke for suggestions regarding data analysis, and G. Hämmerling, B. Arnold and M. Feuerer for discussions. This work was supported by grants from the Helmholtz Association (VH-NG-306) to M.P., the German Research Foundation to M.P. and W.W. (Deutsche

Forschungsgemeinschaft SFB 938 TP K), the Hertie Foundation to W.W. and the Helmholtz Alliance on Systems Biology to S.T. and I.L. T.S. is supported by a DKFZ PhD Program stipend. C.A.O. is supported by a Heidelberg University Medical Faculty Postdoctoral Fellowship.

Author Contributions C.A.O. and U.M.L. contributed equally to this study, designed and performed experiments, analysed data and wrote the paper. F.S. and A.D. analysed protein expression by immunohistochemistry. I.T. cloned constructs and designed experiments. S.T. and I.L. performed nuclear translocation assays. M.O. performed animal experiments. T.S performed immune experiments. L.J. and M.J. performed MR scans. C.L.M. and G.J.G. provided antibodies and designed experiments. D.S. performed and analysed EROD and DRE-luciferase assays. C.L. synthesized the TDO inhibitor. M.W. and W.W. were involved in study design and data interpretation. B.R. analysed microarray data. M.P. and C.A.O. conceptualized the study, interpreted data, designed experiments and wrote the paper. All authors discussed the results and commented on the manuscript.

Author Information Microarray data were deposited in the Gene Expression Omnibus repository (GEO) at www.ncbi.nlm.nih.gov/geo/ under accession number GSE25272. Reprints and permissions information is available at www.nature.com/reprints. The authors declare no competing financial interests. Readers are welcome to comment on the online version of this article at www.nature.com/nature. Correspondence and requests for materials should be addressed to M.P. (m.platten@dkfz-heidelberg.de). 